Théologiques

Théologiques

\title{
Le Message à nos évêques. Une prise de parole, un geste prophétique
}

\section{André Charron}

Volume 16, numéro 1, 2008

Le territoire et le sacré

URI : https://id.erudit.org/iderudit/019188ar

DOI : https://doi.org/10.7202/019188ar

Aller au sommaire du numéro

\section{Éditeur(s)}

Faculté de théologie et de sciences des religions, Université de Montréal

\section{ISSN}

1188-7109 (imprimé)

1492-1413 (numérique)

Découvrir la revue

Citer cet article

Charron, A. (2008). Le Message à nos évêques. Une prise de parole, un geste prophétique. Théologiques, 16(1), 143-161. https://doi.org/10.7202/019188ar
Résumé de l'article

Sous la forme d'un message à leurs évêques à l'occasion de leur visite ad limina à Rome, les supérieurs majeurs des communautés religieuses du Canada ont pris la parole sur la situation de leurs Églises locales. Ils reconnaissent des réussites, signalent des manques, remettent en question des orientations respectant trop peu l'expérience et le discernement des croyants, souhaitent ouverture et débats. Il s'agit là d'un geste prophétique d'éveil aux questions essentielles et d'appel à considérer les personnes dans leur cheminement. Or, applaudi par des laïcs, ce message n’a pas été reçu par les évêques. Cette non-réception a créé l'événement autant que la prise de parole. Les religieux ont osé suggérer d'ouvrir à la discussion des questions même controversées. Ils souhaitent un nécessaire travail d'interprétation de la foi, de la morale et des pratiques dans les conditions culturelles actuelles. 


\title{
Le Message à nos évêques. Une prise de parole, un geste prophétique
}

\author{
André CHARRON* \\ Faculté de théologie et de sciences des religions \\ Université de Montréal
}

Des religieuses et des religieux du Canada, supérieurs majeurs de leurs communautés, se sont exprimés collectivement à l'occasion de la visite ad limina des évêques canadiens à Rome en 2006. La Conférence religieuse canadienne (CRC), qui regroupe ces 230 supérieures et supérieurs majeurs, avait fait la recommandation, à son assemblée générale de 2004, de prendre la parole publiquement dans la société et dans l'Église. Par la décision de son conseil d'administration, elle a choisi de contribuer à un bilan sur l'état de l’Église canadienne en ce moment précis où les évêques du Canada se préparaient à présenter aux congrégations romaines la situation de leurs Églises particulières ${ }^{1}$.

\section{Un message concerté}

Pour être sûr de bien représenter les observations des membres, une large consultation par sondage a été menée auprès de ceux-ci au moyen de questions ouvertes portant sur cinq champs de la réalité ecclésiale : 1) l'Église et la quête de sens, 2) la vie communautaire en Église, 3) la célébration en Église, 4) les solidarités ecclésiales, 5) le prophétisme dans l'Église. Il s'agissait, en chacun de ces champs, A) de reconnaître les réalisations marquantes, B) de signaler les points en souffrance, C) de formuler des souhaits

* André Charron c.s.c. est professeur honoraire de la Faculté de théologie et de sciences des religions de l'Université de Montréal. Au moment des événements, il était supérieur provincial de la Province canadienne des Pères de Sainte-Croix et à ce titre membre de la CRC.

1. Conférence religieuse canadienne (2006). Ce livret broché comprend deux versions de 28 pages, l'une en français, l'autre en anglais. Il est reproduit dans Ambeault (2007, 101-137), ouvrage paru après la rédaction du présent article. 
ou recommandations ${ }^{2}$. Un comité ad hoc a conçu le questionnaire, a dépouillé les réponses et a coordonné la rédaction des résultats. Plus de 2000 réponses à 15 questions ouvertes ont requis un long travail de dépouillement. Une analyse de contenu, rigoureusement conduite en comité, a précédé la synthèse rédactionnelle qui, malgré de nécessaires compressions, a tâché de respecter la substance des propos en y conservant les corrélations et les nuances. Plutôt qu'un texte continu, le format retenu offre finalement un répertoire de 137 énoncés classés par regroupements thématiques.

Le Message à nos évêques présente ainsi les perceptions qu'ont les religieux sur la réalité actuelle de leurs Églises locales. Ils y apportent leurs points de vue avec leur sensibilité propre de religieuses et de religieux pleinement présents à leurs Églises. Engagés sur divers terrains où ils côtoient des gens de toutes conditions, ils répercutent aussi ce que reconnaît, regrette et souhaite le peuple chrétien.

Ce message est adressé aux évêques parce qu'ils sont les pasteurs qui vont faire connaître, lors de leur visite ad limina, les caractéristiques actuelles de leurs Églises locales et les défis auxquels elles ont à faire face. Le message se veut une contribution et donc un appui à cette tâche difficile. Il n'est nullement une charge portée contre les évêques d'ici, ni contre leurs vis-à-vis romains. Il ne comporte pas d'accusation. Il souligne des réussites, il signale des manques, il remet en cause des orientations et des pratiques qui ne relèvent pas seulement du leadership ecclésial mais aussi du membership et d'instances externes comme, par exemple, les médias d'information. Il est solidaire des préoccupations et des souffrances de maints croyants et croyantes d'ici. Doit-on voir ce message comme une interpellation ? Il n'est en rien une apostrophe. Mais, du fait de son expression d'opinion publique claire et percutante, il suscite certes un écho.

\section{Un message diversement reçu}

Or, du côté des destinataires, l'écho n'est pas venu. Les évêques n'ont pas reçu ce message. Ce fut, au sens plein du mot, un acte de non-réception. Cela s'est affiché de plusieurs manières. L'exécutif de la Conférence des évêques catholiques du Canada (CÉCC), pourtant prévenu depuis un an qu'un message allait venir, a ordonné à son secrétariat de ne pas acheminer les publications du Message à l'adresse des évêques; après un mois à dormir sur les tablettes à l'insu de la CRC, les publications ont été récupérées par cette

2. Pour plus de détails, voir Charron (2006). 
dernière, qui a dû les envoyer à chacun des évêques en décembre 2005. Lors de la réunion conjointe des exécutifs de la CÉCC et de la CRC en janvier 2006, les religieux se sont vus opposer une fin de non recevoir. À la réunion plénière de l'Assemblée des évêques catholiques du Québec (AÉCQ) au mois de mars suivant, les rapports avec les religieux sont restés froids. Le président des évêques du Québec, Mgr Gilles Cazabon, a déclaré à la télévision qu'il savait déjà tout ce que les religieux abordaient, qu'il n'avait donc rien à apprendre et qu'il voyait difficilement comment les choses pourraient bouger. Le président des évêques du Canada, Mgr André Gaumond, a confié à des journalistes qu'il doutait de la représentativité des supérieurs. De fait, des évêques ont demandé à des religieuses qui sont à leur service si elles se reconnaissaient dans le Message et ils affirment sans sourciller qu'elles ont répondu non!

La Conférence religieuse canadienne est l'association des supérieurs généraux et des supérieurs provinciaux des religieux et religieuses du Canada et le Message est celui de ces leaders de communautés de vie consacrée. Ceux-ci n'étaient pas tenus de consulter leurs communautés respectives. Si les 22,000 religieuses et religieux du Canada avaient été directement consultés, ou si un sondage scientifique avait été administré auprès d'un échantillon, on peut certes présumer qu'une minorité d'entre eux ne se serait pas reconnue. Mais le Message représente l'opinion d'une nette majorité des supérieurs majeurs qui ont participé à plus de $60 \%$ à la consultation et dont les réponses sont d'une convergence étonnante. Bien entendu, un message collectif ne peut satisfaire tout le monde au même degré; l'un ou l'autre pourrait désirer une formulation plus raffinée de tel ou tel énoncé. Or la confirmation est venue à l'assemblée générale de la CRC de juin 2006: les supérieurs majeurs à l'unanimité ont exprimé leur accord avec la teneur du Message à nos évêques et se sont dits heureux de cette prise de parole.

Manifestement, les évêques ont été surpris, décontenancés, sinon mécontents de ce que les supérieurs aient pris la parole collectivement, ouvertement et sans ménagement. Une fuite dans La Presse du 3 mars 2006 a vite fait de rendre public le contenu quelques jours après la lettre ouverte des 19 prêtres du Forum André Naud sur des questions relatives à l'homosexualité. Les médias en ont fait leurs grands titres et les évêques du Québec ont fini par émettre un communiqué laconique sur les deux affaires, au terme de leur réunion plénière. Ils affirment regretter «d'avoir été entraînés par la force des choses dans une dynamique de confrontation qui risque de durcir les positions ». Ils disent partager des préoccupations mentionnées 
dans le Message de la CRC et ils rappellent qu'il existe déjà des lieux de dialogue dans l'Église: "Nous incitons les membres de l'Église à participer davantage aux divers lieux d'échanges qui existent dans leur milieu. Le dialogue est ouvert. Nous sommes réceptifs à toute suggestion qui favoriserait une meilleure concertation entre toutes les instances de la vie de l'Église ${ }^{3}$. » Mais alors, ils n'ont esquissé aucun geste de concertation avec les religieux avant leur visite à Rome. Ni leur rapport, ni l'allocution de leur président devant le pape n'ont fait mention du Message de la CRC dont l'intention était précisément de les appuyer dans l'analyse de la situation canadienne. Leur secrétaire a informé par la suite qu'il en avait été question à quelques reprises lors des visites faites aux différents dicastères ${ }^{4}$. Depuis lors, le débat sur les questions en cause ne paraît pas avoir avancé 5 .

Beaucoup de laïcs qui ont lu le Message ont conclu qu'il donnait un portrait exact de la situation de l'Église et que c'était là leur pensée et leur sentiment. Ceux du réseau Culture et foi s'y sont retrouvés. Les fidèles de la Communauté Saint-Albert-le-Grand à Montréal ont signé une pétition d'appui. De nombreux courriels envoyés par des religieux à la CRC ont souligné la bouffée d'air frais, la parole prophétique, «le regard bien incarné au cœur des enjeux sociaux et ecclésiaux contemporains ». Un autre

3. Communiqué de l'Assemblée des évêques catholiques du Québec du 9 mars 2006, sur son site Internet.

4. Le rapport présenté à Rome, daté de janvier 2006 (Assemblée des évêques du Québec 2007, 23-71), aurait été rédigé avant la connaissance du document des religieux, d'où son silence, dit-on. Mais l'allocution de Mgr Cazabon du 11 mai 2006 l'ignore aussi complètement (74-78). Manifestement en réponse, le pape Benoît XVI, dans son discours aux évêques du Québec, consacre deux pages sur cinq aux personnes consacrées. Il les invite à accueillir et à diffuser la doctrine de l'Église dans son intégrité et son intégralité $[. .$.$] notamment par une juste interprétation du concile Vatican II, à$ savoir «dans une herméneutique de la réforme, du renouveau dans la continuité de l'unique sujet-Église», concile qui "peut être et devenir toujours davantage une grande force pour le renouveau toujours nécessaire de l'Église » (82s). Somme toute, c'est bien l'impératif dont se réclame le Message des religieux: l'herméneutique de la réforme et la nécessité du renouveau de l'Église.

5. Un comité de travail a été mis sur pied, formé de deux évêques, un francophone et un anglophone, et d'autant de religieux. Il aurait choisi d'échanger sur les conditions d'un dialogue. Or le but du Message n'était pas d'entreprendre un dialogue entre les religieux et les évêques, mais de présenter un ensemble de perceptions et de recommandations sur le service aux chrétiens, de réclamer un dialogue avec des groupes de croyants de la base, voire d'identifier un certain nombre de questions à débattre publiquement. Qui sera mis au courant d'un dialogue en catimini dans le secret d'un tel petit comité ? 
écrit: «Un grand merci à la CRC pour cette prise de position et pour le courage de défendre, sur la place publique et en solidarité avec tant de croyants et de croyantes, cette nécessité d'ouvrir un dialogue franc et réel dans notre Église sur un certain nombre de sujets. Nous sommes plusieurs, soyez certains, à nous reconnaître dans ce document que vous présentez à nos évêques. L'Église d'ici est vivante ${ }^{6}$. » Un autre, en revanche, craint que les religieux se soient isolés au sein de l’Église.

Un avocat des milieux populaires et communautaires, Dominique Boisvert (2006), a lancé une volée de bois vert dans une lettre à ses frères évêques, dénonçant dans les réactions de leurs responsables l'autocensure et la langue de bois qui s'enferme dans le discours institutionnel: "nous n'avons plus de pasteurs à l'écoute de leur peuple mais des officiers prisonniers de leur chaîne de commandement». Il suppose que, si la CRC habituellement discrète a choisi de parler, c'est là un geste de dernier recours. "Comment avez-vous pu tarder à en accuser réception et [...] à vous mettre sincèrement à l'écoute de ce que vos alliés les plus proches se sont sentis obligés de vous écrire à travers cette procédure inusitée ${ }^{7}$ ? ". Le président des Journées sociales du Québec, Guy Paiement s.j., commentait le document de la CRC comme suit dans Le Devoir:

Rarement aura-t-on vu, au Québec, une interpellation aussi radicale que celle-là, venant d'un milieu pourtant avancé en âge, mais nourri par un sentiment d'urgence qui invite à relativiser les problèmes d'intendance pour reprendre le chemin avec les gens. Il faut saluer cette audace des religieuses et des religieux et la laisser nous «travailler» quelque temps avant de la réduire à du déjà connu. Elle a pour nous une saveur d'évangile et des complicités certaines avec tous ceux et celles qui croient qu'un «autre monde est possible» dès ici-bas (Paiement 2006, A-7).

Un diacre permanent, Marc Pepper, écrit au président de la CRC (2006, $13)$ : «Je tenais à vous dire un grand merci pour cette audace évangélique et le sens prophétique de la mission qui vous habite profondément. [...] Merci pour le sérieux avec lequel vous avez proposé des pistes d'avenir qui pourraient devenir le projet pastoral et prophétique de notre Église. » L'historien de l'Église professeur à l'UQAM, Louis Rousseau, soutient en interview ce qui suit.

6. Ces quelques extraits sont publiés dans Charron (2006).

7. Publiée dans la revue Reflets, Province canadienne des Pères de Sainte-Croix, cette «Lettre» fut d'abord diffusée par courrier électronique, le 12 mars 2006. 
Le texte des supérieurs des congrégations religieuses marque un virage dans l'histoire contemporaine de l'Église catholique parce qu'en se retrouvant dans les médias, il a enclenché un débat public. [...] Les membres des communautés religieuses réalisent qu'ils sont en situation de détresse, mais les hautes sphères de l'Église catholique ne s'en inquiètent pas. Le bateau est en train de couler et les officiers frottent leurs habits et vont à la télévision pour dire que ça va bien. Pendant ce temps, l'eau monte dans la cale. Les membres de la Conférence religieuse du Canada le savent très bien et, en écrivant cette lettre, ils ont dit à l'épiscopat de se grouiller, qu'il n'avait pas 25 ans pour régler le problème (Letarte 2006, G-5).

Enfin, on a eu tardivement dans le témoignage de Mgr Gilles Cazabon une part d'explication en ce qui concerne le choc ressenti par les évêques. "Je vais vous parler de ma première réaction. J'ai ressenti en moi de la tristesse et une grande déception. Je m'attendais à ce que les supérieurs majeurs nous parlent surtout de la vie religieuse telle qu'elle est vécue aujourd'hui au Canada pour que nous puissions ensuite, nous qui allions à Rome, la refléter auprès des congrégations romaines. [...] J'ai perçu dans ce message comme une charge " (Claveau 2006, 8). Puis il passe à sa seconde réaction.

J'ai essayé de comprendre comment il se fait que les supérieurs majeurs en viennent à s'exprimer de cette façon. J'ai compris qu'ils ne voulaient pas tellement nous parler de la vie religieuse que nous communiquer leur perception de l'Église canadienne à partir de leurs lieux d'engagement et du caractère prophétique de la vie religieuse. J'ai saisi qu'ils voulaient contribuer ainsi au bilan de la vie ecclésiale au Canada alors que nous partions pour Rome pour la visite ad limina. Et j'ai compris que leur intention était d'agir en appui aux évêques et dans l'esprit de fidélité créatrice avec eux et que leur intention était de favoriser un dialogue en Église (Claveau 2006, 9).

Cette formulation est à ce point le calque de la présentation du président de la CRC, Alain Ambeault c.s.v., qu'il y a lieu de se demander si elle dit vraiment tout d'une attitude qui reste énigmatique.

\section{Un message substantiel}

Quels sont donc les principaux contenus de ce message ? Le document de la CRC offre en effet une matière abondante et substantielle, résultat de la consultation des membres. Il ne s'agit pas ici de réécrire ni d'analyser. Qu'il suffise de rappeler quelques-uns des sujets évoqués. 
- Une Église ouverte au monde, davantage à l'écoute des espoirs et des angoisses des hommes et des femmes pour arriver à offrir une Bonne Nouvelle qui réponde à leur quête de sens.

- En matière d'éthique, une prise en compte de l'expérience des gens, de leur cheminement et de leur croissance, plutôt que l'idéal des principes, la rigidité des normes, le légalisme, l'intransigeance.

- La reconnaissance de la primauté de la personne et l'éclairage des consciences dans le respect de leur liberté fondamentale.

- L'appréciation des expériences, des dires et des initiatives des laïcs. Le poids de leur manque de liberté de parole et de leur perte de confiance en l'Église.

- L'accueil et l'ouverture pour les préoccupations et les valeurs des jeunes générations, l'accompagnement spirituel des adolescents, la garantie d'un engagement financier à l'endroit des mouvements d'action catholique de milieux.

- L'habilitation des chrétiens à verbaliser leur foi, et l'investissement dans les petites communautés et les groupes de partage.

- Dans l'opération de la restructuration des paroisses, la consultation des membres du peuple de Dieu, l'attention aux rapports de proximité, au sens de l'appartenance, à la revitalisation communautaire.

- Des espaces pour les débats. Des lieux d'échange et de dialogue car l'Église institutionnelle s'est refermée considérablement sur elle-même. Promotion du dialogue tout autant sur les grands enjeux de la foi, de la pratique religieuse, de l'action sociale que sur les questions controversées. Acceptation de la pluralité des opinions, d'un niveau raisonnable de dissentiment. Participation des personnes actuellement marginalisées dans l'Église.

- La consultation effective et une véritable coresponsabilité des laïcs dans les décisions qui les concernent.

- La place et le rôle des femmes dans l'Église. Le langage inclusif en liturgie.

- Pour assurer la vie sacramentelle des communautés chrétiennes actuelles et futures, l'ouverture à la discussion en Église et aux voies novatrices du côté des évêques quant à la préparation théologique appropriée pour les laïcs manifestant des habiletés pour le ministère, quant au célibat optionnel pour les prêtres (ordination d'hommes mariés), et à la considération de l'ordination des femmes.

- La pratique du sacrement du pardon avec absolution collective. 
- La formation théologique des prêtres, leur préparation à l'homélie, à l'exercice du leadership spirituel des communautés chrétiennes, l'accessibilité du langage ecclésiastique aux chrétiens d'aujourd'hui, l'acculturation. Le soin dans les rites et une grande attention aux personnes qui ont quitté la pratique religieuse.

- La formation d'agents et agentes en pastorale, catéchèse, théologie, analyse sociale, pédagogie et andragogie, accompagnement spirituel; défi de la nouvelle évangélisation et de l'éducation à la foi chrétienne, des parcours catéchétiques.

- L'autonomie des conférences épiscopales, l'exercice de la collégialité épiscopale, la synodalité des Églises locales. Le retour à la vision pastorale de Vatican II.

- L'engagement effectif des communautés religieuses, en personnel et en soutien financier, dans les milieux de pauvreté et auprès des groupes populaires, en territoires de mission, auprès des aborigènes, dans la promotion des droits humains, la responsabilité écologique et la défense de la justice.

- L'engagement de religieux, religieuses et laïcs formés dans l'accompagnement de personnes en situations particulières: divorces, suicides, itinérances, toxicomanies, maladies terminales, recherche de sens et enjeux de foi.

- La présence et l'action pastorale de notre Église dans les écoles, les hôpitaux, les prisons, auprès des distants, des migrants; la sollicitude caritative des communautés chrétiennes pour les besoins urgents et les services d'entraide: jeunes en détresse, familles monoparentales, assistance aux défavorisés, etc.

- L'accueil de tous les marginalisés de notre monde: femmes, divorcés réengagés, homosexuels, exclus, etc.

- La diffusion des prises de parole et des publications sur les causes humanitaires, la justice et l'écologie auprès des communautés chrétiennes si peu conscientes de la doctrine sociale de l'Église.

- L'audace prophétique de notre Église à rappeler certaines valeurs humaines, sociales et évangéliques alors que sa prise de parole n'est pas toujours bien reçue dans les médias et la collectivité.

\section{Un geste prophétique}

Les religieuses et les religieux se situent, de manière distinctive ou typique, dans la fonction prophétique de la mission de l'Église pour y exercer le 
témoignage d'une vie radicalement engagée, l'éducation à la foi et l'accompagnement spirituel, alors que les évêques et leur presbytérium sont dans la fonction hodégétique de conduite de la communauté chrétienne pour en exercer la gouverne, la direction et l'organisation. Les religieux sont du côté des charismes de libre mouvance, les pasteurs sont du côté des ministères hiérarchiques et des activités de direction de l'institution. Différents, ces types de charismes sont complémentaires dans l'Église communion.

Le prophète, depuis la Bible et le christianisme primitif, répond à la vocation d'être homme de Dieu. Il est le voyant, le messager, le porte-parole de Dieu, qui tâche de communiquer le vrai sens des choses de Dieu. Éveilleur, doté du don de discernement spirituel, ses tâches l'amènent à avertir, exhorter, critiquer, dénoncer. Et encore, le prophète est visionnaire, il anticipe des horizons nouveaux, il prépare le chemin, il provoque la réforme.

Les religieux s'inscrivent dans la lignée du prophétisme chrétien d'abord par la totalité de leur engagement à la suite de Jésus dont ils cherchent à reproduire la forme de vie et le mode d'action. Jésus lui-même s'est identifié avant tout comme prophète, annonciateur, instaurateur et porteur du projet du Règne de Dieu. La substance de la vie consacrée est le cheminement spirituel, l'itinéraire de vie qui s'efforce de témoigner des valeurs du Règne de Dieu. Les religieux, en cela, devaient-ils parler d'eux-mêmes dans le Message à nos évêques ? Ils ont préféré parler plutôt de la situation des gens et de celle de l'Église.

Ceux qui professent la vie consacrée participent en effet à la grande recherche spirituelle du monde moderne. Comme le dit Joan Chittister, grâce à son immersion culturelle, la vie religieuse porte attention aux besoins des gens qui l'entourent, elle se fait le miroir de leurs luttes: «en tout temps et en tout lieu, la personne religieuse qui a fait des questions essentielles de l'humanité le centre de sa vie a toujours été perçue comme une lumière jaillissant des ténèbres spirituelles et comme une gardienne des aspirations fondamentales de l'existence humaine " (1998, 30-31). Ces questions surgissent au cœur du monde séculier: elles concernent la quête de sens, la justice, la paix, l'écologie, la pauvreté, les inégalités, l'oppression, les excès de l'individualisme. Les religieux s'y engagent largement et dans plusieurs énoncés du Message ils éveillent l'Église à ces considérations : que notre Église soit davantage à l'écoute des espoirs et des angoisses des hommes et des femmes de ce temps - spécialement des pauvres et des affligés -, qu'elle retourne à la vision pastorale de Vatican II, qu'elle instaure 
des espaces pour les débats de société concernant par exemple les familles éclatées, les familles reconstituées, la violence conjugale, la surconsommation, le dialogue entre les cultures et les religions, qu'elle soit une Église de l'écoute, du respect, de l'humble recherche de vérité. Oui, ce Message est en cela un geste prophétique d'éveil et d'incitation à réaliser les dispositions du Règne de Dieu. En exhortant à la compassion, en avisant d'accorder la priorité à la considération des personnes et de leur cheminement existentiel, cette prise de parole est prophétique à la manière de l'Évangile.

Le Message a osé parler de contraception, d'homosexualité, de divorce, de protection contre le sida, parce que les gens sont précisément devant ces réalités concrètes. Les religieux, comme toute autre personne, peuvent déplorer que les médias, lorsqu'ils parlent de l'Église et de la religion, portent l'attention exclusivement sur ces questions populaires plutôt que sur la foi, la contribution de la religion à la société, l'humanisme chrétien, l'aide humanitaire de combien d'institutions catholiques, etc. Mais il faut bien reconnaitre que ces questions populaires concernent bien des gens dans la gestion de leur propre existence. Or le Message ne s'oppose pas à la morale sexuelle catholique, mais il en dénonce l'angle de traitement unilatéral, celui d'une perspective essentialiste, idéale et normative et non pas existentielle comme en toute réalité humaine entrant dans l'expérience et dans le cheminement de croissance des personnes.

Geste prophétique aussi, que de parler de la place et du rôle de la femme dans l'Église. Ce thème est d'autant plus récurrent dans le Message, que la CRC est constituée d'une majorité de femmes. Il y a là des observations et des accents très voisins de ce que démontre J. Chittister (1998), quand elle rappelle les résistances de l'Église-institution devant les nouveaux ministères et les nouveaux rôles développés par les religieuses au cours du $\mathrm{XX}^{\mathrm{e}}$ siècle, puis l'impossibilité longtemps pour les femmes d'avoir accès à un diplôme de théologie. "Élargir les limites de l'institution apparaît manifestement comme une fonction de la vie religieuse. C'est ce que les documents officiels appellent la dimension prophétique de la vie religieuse, bien que les fonctionnaires ecclésiastiques lui préfèrent souvent le nom de désobéissance » (307-308).

À l'origine, dit-elle, ce ne sont pas les diocèses qui ont mis sur pied les soupes populaires, les centres de justice et paix, les maisons d'accueil, les centres pour femmes battues, les hospices pour personnes atteintes du sida, les refuges et les centres de spiritualité, mais les sœurs elles-mêmes. [...] Les religieux sont donc par nature des gens qui provoquent des changements. Les 
relations continueront d'être tendues (entre le charisme et l'institution) tant et aussi longtemps que les religieux continueront à faire ce qui doit être fait. [...] La vocation religieuse consiste à sonner l'éveil de l'Église (308-309).

La Bénédictine abonde ensuite dans le féminisme qui s'oppose à toute forme de domination et invite l'Église à mettre en pratique l'égalité qu'elle prêche. Comme Église et comme congrégations, nous ne pouvons nous contenter, dit-elle, «de devenir le volet féminin d'un système patriarcal » (Chittister 1998, 311).

Ce Message à nos évêques n'élude pas les questions controversées qui ont été mises de l'avant dans les résultats du sondage: le célibat optionnel pour les prêtres, l'ordination d'hommes mariés et l'ordination des femmes. Il suggère bien poliment l'ouverture à la discussion de ces questions « dans une recherche continue de la vérité ». Le président de la CRC, Alain Ambeault, l'a bien compris :

Quand on pose des questions sur des points qui sont déjà réglés, souvent la réaction des évêques est de dire qu'ils ne comprennent pas que nous les religieux on évoque des questions comme l'ordination des femmes ou des hommes mariés. Des questions qui sont, semble-t-il, réglées à Rome. Eh bien, le message qu'on a entendu, c'est que ça a beau être réglé à Rome, ce ne l'est pas ici. On dit que ça ne peut pas continuer comme ça (Claveau 2006, 7).

Cette opinion est celle aussi bien des religieux que des laïss. Les évêques d'ici diront probablement que certaines questions ne sont pas de leur ressort et que d'en parler à Rome risquerait la confrontation. Or, précisément dans une visite ad limina, il leur était proposé d'informer les instances romaines que les catholiques canadiens désirent poursuivre les débats. Dans les deux paragraphes qui suivent, voyons de façon plus serrée l'état d'une question supposée fermée pour illustrer la légitimité de la recherche continue.

Au moins une des questions soulevées, le non-accès des femmes à l'ordination, est classée à Rome dans la catégorie des «enseignements définitifs ». Les religieux devaient-ils s'interdire de mentionner ce sujet ? L'affaire est délicate, mais ils ont choisi la transparence et la liberté de pensée dans la foi et dans l'Église. Notons toutefois que leur Message n'est ni une contestation ni une revendication pour l'ordination des femmes, mais le souhait que le débat et la réflexion à peine amorcés soient continués. La catégorie des "enseignements définitifs » est apparue dans le discours romain lors de l'imposition de la Nouvelle profession de foi en 1989. La Société Canadienne de Théologie, réunie en congrès, avait dénoncé alors que trois paragraphes d'inégale portée ayant rapport avec le Magistère soient ajoutés au Credo de 
Nicée-Constantinople et mis au compte d'une profession de foi ${ }^{8}$. Le deuxième paragraphe demandait de "retenir pour vrai tout ce qui concerne la doctrine de la foi ou la morale et est proposé par cette même doctrine de façon définitive ». Le troisième paragraphe demandait "d'adhérer avec un respect religieux de la volonté et de l'intellect aux doctrines énoncées par le Pontife romain ou par le Collège des évêques lorsqu'ils exercent le Magistère authentique, même s'ils n'entendent pas les proclamer par un acte définitif ». Le terme "définitif» y est ambigu et énigmatique: il frôle «l'infaillible» mais il ne l'est pas. Si le pape veut l'infaillible, en effet, il le déclare ex cathedra. Au dire d'André Naud, ce texte de la seconde partie de la profession de foi subdivisée en trois paragraphes est tombé sur l'Église sans qu'évêques ni théologiens n'aient été consultés. Cette Église, "c'est l'Église de ce concile où l'on a tant et tant parlé - espéré ! - de collégialité réelle des évêques et de participation de tous les croyants à la vie et à la pensée qui bouillonnent en elle. [...] C'est aussi l'Église qui arrive mal à définir les libertés qui restent à l'intérieur de la foi, pour tous les croyants, pour les divers pasteurs, pour tous les chercheurs, théologiens, exégètes [...]» $(1989,4-5)$.

Or, voilà que le 22 mai 1994 est publiée la Lettre apostolique Ordinatio sacerdotalis sur l'ordination sacerdotale réservée aux hommes, où le pape déclare «que l'Église n'a en aucune manière le pouvoir de conférer l'ordination à des femmes et que cette position doit être définitivement tenue par tous les fidèles de l'Église ${ }^{9}$ ». Le problème de l'enseignement définitif réapparaît. La réception en fut une de désarroi un peu partout. «On éprouvait énormément de difficulté à concevoir qu'une doctrine d'une si grande portée puisse être imposée comme appartenant au dépôt de la foi sans que soient consultés les évêques, les théologiens et, d'une certaine manière, le peuple fidèle lui-même " (Naud 1996, 103) ${ }^{10}$. Le sens du définitif continue de prêter à confusion. La Congrégation pour la Doctrine de la foi donne une réponse ad dubium: "cette doctrine exige un assentiment définitif [...] parce qu'elle a été proposée infailliblement par le magistère ordinaire et universel ». Dans cette explication la Congrégation va au-delà de la Lettre apostolique; mais elle ne jouit pas du privilège de l'infaillibilité, puisque le charisme de l'infaillibilité pontificale n'est pas transmissible à un organisme

8. Communiqué paru dans Le Devoir, 1er novembre 1989.

9. La Documentation catholique, 19 juin 1994, p. 552.

10. Voir tout le chapitre "Malaises autour du "définitif" ", très documenté et argumenté, p. 73-118. 
du Saint-Siège ${ }^{11}$. D'ailleurs, la Lettre apostolique elle-même ne contient pas un enseignement ex cathedra et l'affirmation de la Congrégation selon laquelle l'ordination réservée aux hommes a été proposée infailliblement n'est pas démontrée. Selon le canon 749,3 « aucune doctrine ne doit être considérée comme définie d'une façon infaillible, à moins que ce ne soit manifeste ". Or trois voies sont indiquées par les documents de l'Église par lesquelles cette vérification pourrait être faite: une consultation universelle des évêques, le consensus universel des théologiens, la commune adhésion des fidèles conformément au canon 750. "À l'évidence, nous sommes devant un enseignement non infaillible qui enseigne quelque chose qu'on peut légitimement estimé incertain » (Naud 1996, 117). Par ailleurs, l'examen exégétique continue: la bibliste canadienne Olivette Genest a écrit récemment $(2006,54)$ : «Ladite clarté des textes bibliques sur l'ordination réservée aux hommes n'arrive pas à remporter l'adhésion. Dans l'état actuel du corpus romain ad hoc, le seul véritable argument qui s'impose, c'est l'argument d'autorité. »

Par leur prise de parole, les religieux n'ont pas la prétention d'accaparer la fonction prophétique, laquelle est celle de toute l'Église comme peuple fidèle. Leurs observations critiques ils les font ici, certes, avec le poids d'un corps constitué et organisé, mais ils les font avant tout en tant que membres engagés de l'Église. Il y a effectivement trop peu de prises de parole dans le peuple de Dieu. Pourtant il doit y avoir une chose telle que l'opinion publique dans l'Église. Pie XII disait, en 1950: «Là où n'apparaît aucune manifestation de l'opinion publique [...] on devrait y voir une maladie de la vie sociale». Puis il le disait de l'Église «car elle est un corps vivant et il manquerait quelque chose à sa vie si l'opinion publique lui faisait défaut, défaut dont le blâme retomberait sur les pasteurs et sur les fidèles ${ }^{12}{ }$. Les religieux font donc une bonne chose en prenant la parole publiquement. Et s'ils peuvent aller jusqu'au dissentiment sur certains points, c'est de façon loyale et responsable, comme le suggère Gérald Arbuckle (2000), pour qui il n'y a pas de changement constructif, même en Église, sans qu'il n'y ait une certaine forme de dissentiment - particulièrement en opposition à la culture de restauration d'accent préconciliaire - et pour qui la réponse aux défis pastoraux de notre monde requiert des personnalités prophétiques, des "gens du bond en avant ${ }^{13}$ ».

11. Voir Naud $(1996,108)$, avec référence au canoniste Ladislas Orsy.

12. Dans La Documentation catholique, 1964, 12 mars 1950, p. 327.

13. Je n'aime pas le terme refonder. On ne fonde pas deux fois quoi que ce soit! Mieux vaudrait dire réformer, transformer, faire renaître, relancer, repartir, renouveler, revitaliser. 
Le Concile Vatican II veut des laïcs "qu'ils s'ouvrent aux pasteurs de leurs besoins et de leurs vœux avec toute la liberté qui convienne à des fils de Dieu et à des frères dans le Christ. Dans la mesure de leurs connaissances, de leurs compétences et de leur rang, ils ont la faculté et même parfois le devoir de manifester leur sentiment en ce qui concerne le bien de l'Église » (LG 37). Le Droit canonique entérine cette perspective en stipulant que les fidèles ont le droit et même parfois le devoir de donner aux pasteurs leur opinion sur ce qui touche le bien de l'Église et de la faire connaître aux autres fidèles (c 212,3). Le concile le dit encore ailleurs, notamment dans le cadre de la paroisse (AL 10).

Les références canadiennes ne manquent pas non plus. Plusieurs rapports fondés sur de larges consultations ont proposé, de décennie en décennie, depuis le Rapport Dumont, la circulation de la parole, la concertation et le débat en Église sur les réalités de la foi, de la morale et des pratiques. De nombreux fidèles éprouvent en effet le besoin de rechercher ensemble le vrai sens chrétien en situation aujourd'hui, l'interprétation des croyances, ainsi que des mots pour dire ces croyances dans la culture. Ne serait-ce pas là l'expression actualisée et continue du sensus fidei fidelium, du sens commun de la foi des fidèles, cette faculté d'intelligence de la foi, cette puissance de discernement chrétien appartenant au corps des croyants ? De portée plus globale, un document sur l'exercice de la synodalité dans la vie courante et les activités de l'Église a été conçu et publié par le Comité de théologie de l'Assemblée des évêques du Québec. La pratique de la synodalité, à la fois très nouvelle et très ancienne, s'ajuste à l'esprit démocratique contemporain tout comme aux manières de faire des assemblées plénières délibérantes du christianisme primitif. L'exercice de cette concertation et de cette responsabilité commune, qui opère organiquement selon la diversité des charismes et des fonctions, prend charge des affaires de l'Église locale. D'où l'importance de la circulation des idées et des questions, de la libre prise de parole, de l'expression d'opinion, de la discussion et du dialogue, et même de la participation au processus de décision. Ainsi l'affirme le Comité de théologie $(2000,42)$ à propos des membres actifs de la pastorale de l'Église: "Il faut donc une réelle pratique de communication entre les acteurs. Il peut être requis d'ouvrir des débats sur les grands enjeux humains, sociaux et ecclésiaux. [...] On arrivera ainsi à une solide pratique de discernement communautaire, en étant attentif non seulement au sens évangélique mais aussi aux signes des temps dans les conditions réelles du monde actuel. » Le pasteur, est-il écrit plus loin, fera bien de se rappeler la 
pratique synodale d'illustres prédécesseurs (44): c'est «d'accord avec toute l'Église que les apôtres et les anciens décidèrent (Ac 15,22). » La venue du Message à nos évêques de la CRC n'est pas fortuite.

\section{En guise de conclusion}

Les religieux canadiens ont l'Église en haute estime et ils s'y dévouent grandement. Ils savent reconnaître ce qui s'y fait de pertinent et d'audacieux. Mais ils s'inquiètent de la perte de crédibilité de l'Église-institution dans leur culture. Ils déplorent le décrochage de trop de gens. Ils souffrent des effets dissuasifs qu'a ce recul de l'Église sur les vocations à la vie consacrée ellemême. Ils désirent une relance sur les chemins de la vie et la revitalisation des communautés de foi.

Un observateur externe à la CRC, le père Guy Paiement, a bien évalué le Message à nos évêques:

Le document insiste avec raison sur deux conditions fondamentales qui rendront possibles la vitalité et le développement (des activités ecclésiales). La première, c'est la conviction qu'il faut respecter l'expérience et le discernement des croyantes et des croyants et que des lieux de débat sont indispensables pour leur donner corps. Ils sont actuellement raréfiés, alors que la participation publique aux décisions s'impose de plus en plus dans nos sociétés et façonne notre sensibilité. La seconde, c'est le rappel que l'Église du Québec ou du Canada ne sont pas de simples succursales d'un Wal-Mart romain et que les responsables d'ici devraient avoir le courage de se tenir debout devant les officines romaines. Il y a là deux conditions pour redonner sans doute de la crédibilité aux responsables. Mais on peut y voir d'abord une exigence proprement théologique si, du moins, on croit incontournable d'être attentif à ce que l'Esprit dit aux Églises (Paiement 2006, A-7).

Ces deux conditions ressortent en effet du document. Mais elles s'imposent encore davantage en regard de l'ensemble de l'événement constitué par le message des religieux reflétant l'opinion de bien des fidèles qui confirment s'y reconnaître, sa non-réception par apparemment une bonne part des évêques canadiens et la visite ad limina dont l'objectif premier est de présenter l'état actuel de l'Église en notre pays.

Lors d'une entrevue au micro de Radio Ville-Marie le 10 mars 2006, au beau milieu de la controverse, j'avais avancé que nos évêques d'ici devraient présumer que le pape Benoît XVI saurait accueillir une présentation franche et libre avec plus d'ouverture et d'intelligence pastorale qu'ils ne l'appréhendaient par leur attitude frileuse et tout enveloppée. J'avais 
cité un extrait de son discours improvisé au clergé de Rome quelques jours auparavant:

Vous avez souligné que, au niveau charismatique, des femmes ont fait tellement, j'oserais dire pour le gouvernement de l'Église, en commençant par les religieuses, par les sœurs des grands Pères de l'Église comme saint Ambroise, jusqu'aux grandes dames du Moyen-Âge - sainte Hildegarde, sainte Catherine de Sienne et sainte Thérèse d'Avila - et jusqu'à Mère Teresa. Je dirais que ce secteur charismatique se distingue certainement du secteur ministériel au sens strict du terme, mais c'est une participation véritable et profonde au gouvernement de l'Église. [...] Comment pourrait-on imaginer le gouvernement de l'Église sans cette contribution qui devient parfois très visible, comme lorsque sainte Hildegarde critique les évêques, ou comme lorsque sainte Brigitte et sainte Catherine de Sienne admonestent les papes et obtiennent leur retour à Rome ? C'est toujours un facteur déterminant, sans lequel l'Église ne peut pas vivre ${ }^{14}$.

Toute proportion gardée, l'occasion était trop belle pour ne pas souligner la coïncidence.

Or Gilles Routhier (2007) vient de publier, suite à l'événement occasionné par le message des religieux, la lettre des prêtres du Forum André Naud et celle de 42 laïcs de huit diocèses du Québec sur des sujets dont l'enjeu est le même, un article sur l'actualisation du "droit de remontrance". Il s'agit d'un droit qui fait partie d'une longue tradition de l'Église, bien qu'il n'ait pas été retenu lors des deux codifications récentes du droit canonique. Le droit de remontrance est le droit d'en «remontrer» au pape en raison de la tension entre un enseignement universel et les situations locales: «il affirme le droit reconnu à un évêque d'interpeller (faire une réprimande) l'autorité romaine qui a porté une loi qui blesse son Église du fait qu'elle est mal accordée aux situations locales » (Routhier 2007, 317). Pour Routhier, l'enjeu sous-jacent au droit de remontrance est bien présent dans les messages en cause :

Ce qui est demandé aux évêques, c'est de présenter à Rome, notamment aux congrégations de la curie, les profonds malaises des fidèles à la lecture de certains enseignements formulés ou libellés comme ils le sont. Ces fidèles, prêtres ou laïcs, appartenant ou pas à l'état religieux, ont conscience que leurs évêques ont une action à poser dans ce cas. Leurs interventions sont en fait rattachées à la visite ad limina de leurs évêques (316-317).

14. Extrait du discours de Benoît XVI du 2 mars 2006 transmis par l'agence Zenit.org le 7 mars suivant: <http://www.zenit.org/article-12315?l=french>. 
À tout le moins de manière analogique (car le droit de remontrance est au sens strict un droit épiscopal), les prises de parole invitaient à communiquer aux autorités romaines les «remontrances" des fidèles de nos Églises. Il était demandé aux évêques d'être non pas seulement les porteparole de l'Église de Rome au Canada, mais aussi les porte-parole des Églises du Québec et du Canada à Rome. Cette responsabilité épiscopale qui doit jouer dans les deux sens n'est pas souvent appliquée. Entendons bien: les religieux n'ont jamais voulu adresser des réprimandes à leurs évêques ni aux autorités romaines, mais assurément il y avait de la remontrance au sens canonique du terme dans leur message, cette autre modalité de la fonction critique en Église.

Mais là n'était pas l'intention première du Message. On ne saurait oublier que le tiers en volume de ce texte est une reconnaissance des réalisations encourageantes de nos Églises locales et qu'une bonne partie des regrets puis des souhaits émis sont des interpellations au peuple chrétien et à ses ministres pour plus d'exigence, de compétence et de pertinence. La prise de parole a été une expression d'opinion, un exercice de transparence, une exhortation animée d'un sentiment d'urgence. Elle sonne le réveil sur des points essentiels: la considération des personnes et le nécessaire travail d'interprétation des réalités de la foi, de la morale et des pratiques dans les conditions de la culture actuelle, autrement dit, la compréhension et la pratique du sens chrétien dans la dramatique humaine et existentielle d'aujourd'hui. Elle est un vibrant appel au débat, à la discussion et au discernement communautaires.

\section{Références}

Ambeault, A. (2007), Autopsie d'un débat avorté, Novalis, Montréal.

Arbuckle, G. (2000), Refonder l'Église. Dissentiment et leadership, Montréal, Bellarmin.

Assemblée des Évêques Catholiques du QuéBec (2007), Bâtir l'Église de Dieu qui est au Québec. La visite ad limina des évêques du Québec, Montréal, Fides, (L'Église aux quatre vents).

Boisvert, D. (2006), "Lettre à mes frères évêques ", Reflets, 189, p. 11-12.

Charron, A. (2006), «Le Message à nos évêques: la genèse et l'esprit d'une prise de parole", Bulletin CRC, 3/1, p. 6-7.

ChitTister, J. (1998), Le feu sous les cendres. Une spiritualité pour la vie religieuse contemporaine, Montréal, Bellarmin. 
Claveau, P. (2006/09) «Le message de la CRC aux évêques: un point de discorde ", Présence Magazine, 116, p. 7-9.

Comité De théologie de l'Assemblée des évêQues Du Québec (2000), Vers l'exercice de la synodalité dans nos églises, Montréal, Fides (L'Église aux quatre vents).

CONFÉRENCE RELIGIEUSE CANADIENNE (2006), Message à nos évêques à l'occasion de leur visite ad limina 2006, CRC, Montréal.

GENEST, O. (2006), «Évaluation des fondements bibliques invoqués contre l'accès des femmes au ministère sacerdotal dans l'Église catholique", Colloque Femmes et hommes en Église, Parvis, Hors-série 15, p. 49-54.

Letarte, M. (2006), «Une Église en crise », Le Devoir, 15 avril, G-5.

Naud, A. (1989), Devant la nouvelle Profession de foi et le Serment de fidélité, Montréal, Fides (Débats de l’Église 1).

(1996), Un aggiornamento et son éclipse. La liberté de pensée dans la foi et dans l'Église à Vatican II et aujourd'hui, Montréal, Fides.

Paiement, G. (2006), «Du moraliste à la sage-femme », Le Devoir, 13 mars, A-7.

Pepper, M. (2006), «Votre lettre comme une visitation », Reflets, 189, p. 13.

Routhier, G. (2007), "Les "remontrances" adressées aux évêques du Québec: expression contemporaine d'un droit ancien ?", Science et Esprit, 59/2-3, p. 309-332.

\section{Résumé}

Sous la forme d'un message à leurs évêques à l'occasion de leur visite ad limina à Rome, les supérieurs majeurs des communautés religieuses du Canada ont pris la parole sur la situation de leurs Églises locales. Ils reconnaissent des réussites, signalent des manques, remettent en question des orientations respectant trop peu l'expérience et le discernement des croyants, souhaitent ouverture et débats. Il s'agit là d'un geste prophétique d'éveil aux questions essentielles et d'appel à considérer les personnes dans leur cheminement. Or, applaudi par des laïcs, ce message n'a pas été reçu par les évêques. Cette non-réception a créé l'événement autant que la prise de parole. Les religieux ont osé suggérer d'ouvrir à la discussion des questions même controversées. Ils souhaitent un nécessaire travail d'interprétation de la foi, de la morale et des pratiques dans les conditions culturelles actuelles. 


\section{Abstract}

Through a message addressed to their bishops during an ad limina meeting in Rome, the superiors of Canada's religious communities expressed their concerns regarding the situation of their local Churches. They pointed out some strengths and weaknesses, questioned the fact that some decisions are not taken according to the believers' discernment, and asked for more openness and consultation. This represents a prophetic action bringing forward central questions and calling the Church to pay attention to people's life of faith. But the Message was not heard by the bishops, even if it was praised by the laity. That, as much as the decision to speak out, is significant: the religious communities dared to suggest that some controversial questions should be open to discussion. They are hoping for a necessary interpretive work on faith, morals, and customs within the current cultural setting. 